\title{
Differential equations of divergence form in separable Musielak-Orlicz-Sobolev spaces
}

\author{
Ge Dong ${ }^{1,2+}$ and Xiaochun Fang ${ }^{1 *+}$
}

${ }^{\text {"Correspondence: }}$ xfang@tongji.edu.cn

${ }^{1}$ Department of Mathematics, Tongji University, Siping Road, Shanghai, 200092, China Full list of author information is available at the end of the article ${ }^{\dagger}$ Equal contributors

\begin{abstract}
In this paper, we study the existence of weak solutions for differential equations of divergence form

$$
-\operatorname{div}\left(a_{1}(x, D u)\right)+a_{0}(x, u)=f(x, u, D u),
$$

in $\Omega$ coupled with a Dirichlet or Neumann boundary condition in separable Musielak-Orlicz-Sobolev spaces where $a_{1}$ satisfies the growth condition, the coercive condition, and the monotone condition, and $a_{0}$ satisfies the growth condition without any coercive condition or monotone condition. The right-hand side $f: \Omega \times \mathbb{R} \times \mathbb{R}^{N} \rightarrow \mathbb{R}$ is a Carathéodory function satisfying a growth condition dependent on the solution $u$ and its gradient $D u$. We prove the existence of weak solutions by using a linear functional analysis method. Some sufficient conditions guarantee the existence enclosure of weak solutions between sub- and supersolutions. Our method does not require any reflexivity of the Musielak-Orlicz-Sobolev spaces.
\end{abstract}

Keywords: separable Musielak-Orlicz-Sobolev spaces; differential equation; sub-supersolution

\section{Introduction}

Let $\Omega \subset \mathbb{R}^{N}$ be a bounded domain with Lipschitz boundary. Le [1] established a subsupersolution method for variational inequalities with Leray-Lions operators in Sobolev spaces with variable exponents. Following [1], Fan [2] established a sub-supersolution method for the differential equations of divergence form

$$
-\operatorname{div}\left(a_{1}(x, D u)\right)+a_{0}(x, u)=f(x, u)
$$

in $\Omega$ coupled with Neumann or Dirichlet boundary condition in reflexive Musielak-OrliczSobolev spaces $W_{0}^{1} L_{\Phi}(\Omega)$. Here $a_{1}$ and $a_{0}$ are supposed to satisfy growth conditions, coercive conditions, and monotone conditions, that is,

$$
\begin{aligned}
& \left|a_{1}(x, \xi)\right| \leq b_{1} \varphi(x,|\xi|)+g(x), \\
& a_{1}(x, \xi) \xi \geq b_{2} \Phi(x,|\xi|)-h(x), \\
& {\left[a_{1}(x, \xi)-a_{1}(x, \eta)\right](\xi-\eta) \geq 0,}
\end{aligned}
$$

(c) 2016 Dong and Fang. This article is distributed under the terms of the Creative Commons Attribution 4.0 International License (http://creativecommons.org/licenses/by/4.0/), which permits unrestricted use, distribution, and reproduction in any medium, provided you give appropriate credit to the original author(s) and the source, provide a link to the Creative Commons license, and indicate if changes were made. 
and

$$
\begin{aligned}
& \left|a_{0}(x, t)\right| \leq b_{1} \varphi(x,|t|)+g(x), \\
& a_{0}(x, t) t \geq b_{2} \Phi(x,|t|)-h(x), \\
& {\left[a_{0}(x, s)-a_{0}(x, t)\right](s-t) \geq 0,}
\end{aligned}
$$

for $x \in \Omega, s, t \in \mathbb{R}$ and $\xi, \eta \in \mathbb{R}^{N}$, where $b_{1}, b_{2}>0, g \in E_{\bar{\Phi}}(\Omega), g \geq 0, h \in L^{1}(\Omega)$, and $h \geq 0$. The right-hand side $f: \Omega \times \mathbb{R} \rightarrow \mathbb{R}$ is a Carathéodory function.

Liu et al. [3] proved the existence of weak solutions for (1.1) with $a_{0}=0$ in reflexive Musielak-Orlicz-Sobolev spaces.

However, there exist some nonreflexive Musielak-Orlicz-Sobolev spaces. For example, let $\Phi(x, t)=\left(1+\frac{t}{p(x)}\right) \ln \left(1+\frac{t}{p(x)}\right)-\frac{t}{p(x)}$, for $x \in \Omega$ and $t>0$, where $p: \Omega \rightarrow \mathbb{R}$ is a measurable function such that $1<p_{-}:=\inf _{x \in \Omega} p(x) \leq p(x) \leq p_{+}:=\sup _{x \in \Omega} p(x)<+\infty$. Then the Musielak-Orlicz-Sobolev space $W^{1} L_{\Phi}(\Omega)$ is separable and nonreflexive.

The purpose of this paper is to weaken the restriction of reflexivity of the MusielakOrlicz spaces in [2] and study the existence of solutions for the following nonlinear problem:

$$
-\operatorname{div}\left(a_{1}(x, D u)\right)+a_{0}(x, u)=f(x, u, D u),
$$

in $\Omega$ coupled with Dirichlet or Neumann boundary condition, where $a_{1}$ satisfies the growth condition, the coercive condition, and the monotone condition, and $a_{0}$ satisfies the growth condition without any coercive condition or monotone condition. The righthand side $f: \Omega \times \mathbb{R} \times \mathbb{R}^{N} \rightarrow \mathbb{R}$ is a Carathéodory function satisfying a growth condition dependent on the solution $u$ and its gradient $D u$.

One needs the following coercive condition of $\Phi$ in [2]:

$$
\Phi(x, \alpha u) \geq \alpha G(\alpha) \Phi(x, u), \quad \text { for } x \in \Omega, t \in \mathbb{R} \text { and } \alpha>0,
$$

where $G:(0,+\infty) \rightarrow \mathbb{R}$ is a function such that $G(\alpha) \rightarrow+\infty$ as $\alpha \rightarrow+\infty$. We will point out that the condition (1.9) can be omitted.

This paper is organized as follows: Section 2 contains some preliminaries and some technical lemmas which will be needed. We establish some basic properties for MusielakOrlicz functions and some necessary and sufficient conditions for Musielak-Orlicz functions satisfying the $\Delta_{2}$ condition. In Section 3, we establish a linear functional analysis method for differential equations of divergence form to prove the existence of weak solutions for (1.8) with Dirichlet boundary or Neumann boundary condition in separable Musielak-Orlicz-Sobolev spaces. We give the enclosure of weak solutions between suband supersolutions by using a sub-supersolution method. Our method does not require any monotonicity or coercivity of $a_{0}$. We point out that the coercive condition (1.9) of $\Phi$ can be omitted because of the reflexivity of the Musielak-Orlicz-Sobolev spaces in [2].

We refer to some results of sub-supersolution methods for variational inequalities and the existence of solutions for differential equations studied in variable exponent Sobolev or Orlicz-Sobolev spaces (see, e.g., [4-11]). For some results we also refer to [12-14].

In this paper, we always assume that $\Omega \subset \mathbb{R}^{N}$ is a bounded domain with Lipschitz boundary and denote by $L^{0}(\Omega)$ the set of all real measurable functions defined on $\Omega$. 


\section{Preliminaries}

Now we list briefly some definitions and facts about Musielak-Orlicz-Sobolev spaces; for more details see $[2,15,16]$, and [17].

A real function $\Phi$ defined on $\Omega \times \mathbb{R}_{+}$, where $\mathbb{R}_{+}=[0,+\infty)$, will be said a generalized $N$ function (i.e. a Musielak-Orlicz function), denoted by $\Phi \in N(\Omega)$, if it satisfies the following conditions:

(i) $\Phi(x, u)$ is an $N$-function of the variable $u \geq 0$ for every $x \in \Omega$, i.e. is a convex, nondecreasing, continuous function of $u$ such that $\Phi(x, 0)=0, \Phi(x, u)>0$ for $u>0$, and we have the conditions

$$
\lim _{u \rightarrow 0^{+}} \sup _{x \in \Omega} \frac{\Phi(x, u)}{u}=0, \quad \lim _{u \rightarrow+\infty} \inf _{x \in \Omega} \frac{\Phi(x, u)}{u}=+\infty .
$$

(ii) $\Phi(x, u)$ is a measurable function of $x$ for all $u \geq 0$.

Equivalently, $\Phi$ admits the representation

$$
\Phi(x, u)=\int_{0}^{u} \varphi(x, \tau) d \tau,
$$

where $\varphi(x, u)$ is the right-hand derivative of $\Phi(x, \cdot)$ at $u$, for a fixed $x \in \Omega$ and all $u \geq 0$. Then for every $x \in \Omega, \varphi(x, \tau)$ is a right-continuous and nondecreasing function of $\tau \geq 0$, $\varphi(x, 0)=0, \varphi(x, \tau)>0$ for $\tau>0$, and $\lim _{u \rightarrow+\infty} \inf _{x \in \Omega} \varphi(x, \tau)=+\infty$.

Let $\Phi \in N(\Omega)$, then $\Phi(x, u) \leq u \varphi(x, u) \leq \Phi(x, 2 u)$, for $x \in \Omega, u \geq 0$.

The complementary function $\bar{\Phi}$ to a Musielak-Orlicz function $\Phi$ is defined as follows:

$$
\bar{\Phi}(x, v)=\sup _{u \geq 0}\{u v-\Phi(x, u)\}, \quad \text { for all } v \geq 0, x \in \Omega .
$$

Then $\bar{\Phi}$ is a Musielak-Orlicz function and $\Phi$ is also the complementary function to $\bar{\Phi}$. Equivalently, $\bar{\Phi}$ admits the representation

$$
\bar{\Phi}(x, v)=\int_{0}^{v} \phi(x, \sigma) d \sigma,
$$

where $\phi$ is given by

$$
\phi(x, \sigma)=\sup \{\tau: \varphi(x, \tau) \leq \sigma\}, \quad \text { for all } x \in \Omega \text {. }
$$

Similar to the proof in [18], we can deduce that

$$
\phi(x, \varphi(x, u)) \geq u, \quad \varphi(x, \phi(x, v)) \geq v, \quad \text { for } u \geq 0, v \geq 0 \text { and } x \in \Omega,
$$

and

$$
\begin{aligned}
& \phi(x, \varphi(x, u)-\varepsilon) \leq u, \quad \text { for } u \geq 0,0<\varepsilon \leq \varphi(x, u) \text { and } x \in \Omega, \\
& \varphi(x, \phi(x, v)-\varepsilon) \leq v, \quad \text { for } v \geq 0,0<\varepsilon \leq \phi(x, v) \text { and } x \in \Omega .
\end{aligned}
$$

For $\Phi \in N(\Omega)$, the following inequality is called the Young inequality:

$$
u v \leq \Phi(x, u)+\Phi(x, v), \quad \text { for all } u, v \geq 0, x \in \Omega
$$


and the equality holds if and only if $u=\phi(x, v)$ or $v=\varphi(x, u)$, i.e.

$$
u \varphi(x, u)=\Phi(x, u)+\bar{\Phi}(x, \varphi(x, u)), \quad \phi(x, v) v=\Phi(x, \phi(x, v))+\bar{\Phi}(x, v)
$$

Let $\Phi \in N(\Omega)$. $\Phi$ is said to satisfy the $\Delta_{2}$ condition $\left(\Phi \in \Delta_{2}\right.$, for short), if there exist a positive constant $K>1$ and a nonnegative function $h \in L^{1}(\Omega)$ such that

$$
\Phi(x, 2 u) \leq K \Phi(x, u)+h(x), \quad \text { for all } u \geq 0 \text { and a.e. } x \in \Omega
$$

Clearly, by the proof of Proposition 1.3(6) in [2], if $\Phi \in \Delta_{2}$, then there exist $K>1$ and a nonnegative function $h \in L^{1}(\Omega)$ such that

$$
\bar{\Phi}(x, \varphi(x, u)) \leq(K-1) \Phi(x, u)+h(x), \quad \text { for all } u \geq 0 \text { and a.e. } x \in \Omega
$$

For each $x \in \Omega$, the inverse function of $\Phi(x, \cdot)$ is denoted by $\Phi^{-1}(x, \cdot)$, i.e.

$$
\Phi^{-1}(x, \Phi(x, u))=\Phi\left(x, \Phi^{-1}(x, u)\right)=u, \quad \text { for } u \geq 0 .
$$

Let $\Psi, \Upsilon \in N(\Omega) . \Psi \preceq \Upsilon$ means that $\Psi$ is weaker than $\Upsilon$, i.e., there exist positive constants $K_{1}, K_{2}$ and a nonnegative function $h_{1} \in L^{1}(\Omega)$ such that

$$
\Psi(x, u) \leq K_{1} \Upsilon\left(x, K_{2} u\right)+h_{1}(x), \quad \text { for all } u \geq 0 \text { and a.e. } x \in \Omega
$$

$\Phi$ is called locally integrable, if $\int_{\Omega} \Phi(x, u) d x<\infty$ for every $u>0$.

The following assumptions will be used.

$\left(\Phi_{1}\right) \inf _{x \in \Omega} \Phi(x, 1)=c_{1}>0$.

$\left(\Phi_{2}\right)$ For every $t_{0}>0$ there exists $c=c\left(t_{0}\right)>0$ such that

$$
\inf _{x \in \Omega} \frac{\Phi(x, t)}{t} \geq c
$$

and

$$
\inf _{x \in \Omega} \frac{\bar{\Phi}(x, t)}{t} \geq c
$$

for all $t \geq t_{0}$.

Obviously, (2.10) implies $\left(\Phi_{1}\right)$.

Let $\Phi \in N(\Omega)$. The Musielak-Orlicz space (i.e. the generalized Orlicz space) $L_{\Phi}(\Omega)$ is defined by

$$
L_{\Phi}(\Omega)=\left\{u \in L^{0}(\Omega): \int_{\Omega} \Phi\left(x, \frac{|u(x)|}{\lambda}\right) d x<\infty \text {, for some } \lambda>0\right\} \text {, }
$$

with the (Luxemburg) norm

$$
\|u\|_{\Phi}=\inf \left\{\lambda>0: \int_{\Omega} \Phi\left(x, \frac{|u(x)|}{\lambda}\right) d x \leq 1\right\}
$$


Moreover, the set

$$
K_{\Phi}(\Omega)=\left\{u \in L^{0}(\Omega): \int_{\Omega} \Phi(x,|u(x)|) d x<\infty\right\},
$$

will be called the Musielak-Orlicz class (i.e. the generalized Orlicz class). A function $u \in$ $L^{0}(\Omega)$ will be called a finite element of $L_{\Phi}(\Omega)$, if $\lambda u \in K_{\Phi}(\Omega)$ for every $\lambda>0$. The space of all finite elements of $L^{0}(\Omega)$ will be denoted by $E_{\Phi}(\Omega)$. Then $K_{\Phi}(\Omega)$ is a convex subset of $L_{\Phi}(\Omega), L_{\Phi}(\Omega)$ is the smallest vector subspace of $L^{0}(\Omega)$ containing $K_{\Phi}(\Omega)$, and $E_{\Phi}(\Omega)$ is the largest vector subspace of $L^{0}(\Omega)$ contained in $K_{\Phi}(\Omega)$.

If $\Phi$ is locally integrable, then $E_{\Phi}(\Omega)$ is a separable space, and $E_{\Phi}(\Omega)=K_{\Phi}(\Omega)=L_{\Phi}(\Omega)$ if and only if $\Phi \in \Delta_{2}$.

If $\Phi$ is locally integrable and satisfy $(2.10)$, then $\left(E_{\Phi}(\Omega)\right)^{*}=L_{\bar{\Phi}}(\Omega)$. Moreover, if $\bar{\Phi}$ is locally integrable satisfying (2.11), and $\Phi, \bar{\Phi} \in \Delta_{2}$, then $L_{\Phi}(\Omega)$ is reflexive.

The Musielak-Orlicz-Sobolev space $W^{1} L_{\Phi}(\Omega)$ is defined by

$$
W^{1} L_{\Phi}(\Omega)=\left\{u \in L_{\Phi}(\Omega): \forall|\alpha| \leq 1, D^{\alpha} u \in L_{\Phi}(\Omega)\right\}
$$

where $\alpha=\left(\alpha_{1}, \ldots, \alpha_{N}\right)$ with nonnegative integers $\alpha_{i}, i=1, \ldots, N,|\alpha|=\left|\alpha_{1}\right|+\left|\alpha_{2}\right|+\cdots+\left|\alpha_{N}\right|$ and $D^{\alpha} u$ denote the distributional derivatives.

Let

$$
\varrho_{\Phi}(u)=\sum_{|\alpha| \leq 1} \int_{\Omega} \Phi\left(x,\left|D^{\alpha} u(x)\right|\right) d x \quad \text { and } \quad\|u\|_{\Phi, \Omega}=\inf \left\{\lambda>0: \varrho_{\Phi}\left(\frac{u}{\lambda}\right) \leq 1\right\}
$$

for $u \in W^{1} L_{\Phi}(\Omega) . \varrho_{\Phi}(u)$ is a convex modular and $\|u\|_{\Phi, \Omega}$ is a norm on $W^{1} L_{\Phi}(\Omega)$, respectively. The pair $\left(W^{1} L_{\Phi}(\Omega),\|u\|_{\Phi, \Omega}\right)$ is a Banach space if $\Phi$ is locally integrable and satisfies $\left(\Phi_{1}\right)$.

Taking $\Phi(x, u)=\Phi(u), W^{1} L_{\Phi}(\Omega)$ is the Orlicz-Sobolev space. Taking $\Phi(x,|u|)=|u|^{p(x)}$, $W^{1} L_{\Phi}(\Omega)$ is the variable exponent Sobolev space $W^{1, p(\cdot)}(\Omega)$.

It is easy to see that

$$
W^{1} L_{\Phi}(\Omega)=\left\{u \in L_{\Phi}(\Omega):|D u| \in L_{\Phi}(\Omega)\right\} .
$$

Denote $\|D u\|_{\Phi}=\|\mid D u\|_{\Phi}$ and $\|u\|_{1, \Phi}=\|u\|_{\Phi}+\|D u\|_{\Phi}$. Then $\|u\|_{1, \Phi}$ and $\|u\|_{\Phi, \Omega}$ are two equivalent norms.

The space $W^{1} L_{\Phi}(\Omega)$ will always be identified to a subspace of the product $\prod_{|\alpha| \leq 1} L_{\Phi}(\Omega)=$ $\prod L_{\Phi}$; this subspace is $\sigma\left(\prod L_{\Phi}, \prod E_{\bar{\Phi}}\right)$ closed. Let $W_{0}^{1} L_{\Phi}(\Omega)$ be the $\sigma\left(\prod L_{\Phi}, \prod E_{\bar{\Phi}}\right)$ closure of the Schwartz space $\mathcal{D}(\Omega)$ in $W^{1} L_{\Phi}(\Omega)$.

Let $W^{1} E_{\Phi}(\Omega)=\left\{u \in E_{\Phi}(\Omega): \forall|\alpha| \leq 1, D^{\alpha} u \in E_{\Phi}(\Omega)\right\}$, and $W_{0}^{1} E_{\Phi}(\Omega)$ is the (norm) closure of $\mathcal{D}(\Omega)$ in $W^{1} L_{\Phi}(\Omega)$.

The proof of the following lemma is similar to [19].

Lemma 2.1 Let meas $\Omega$ be bounded, $\Phi \in N(\Omega)$, and $\varphi$ is the right-hand derivative of $\Phi$. Then

$$
\frac{\int_{\Omega} \varphi(x,|D u|)|D u| d x}{\int_{\Omega}|D u| d x} \rightarrow+\infty, \quad \text { if } \int_{\Omega}|D u| d x \rightarrow+\infty .
$$


Proof Let us assume that there is a sequence $\left\{u_{n}\right\}$ with $\int_{\Omega}\left|D u_{n}(x)\right| d x \rightarrow+\infty$ and $K_{0}<\infty$ such that

$$
\frac{\int_{\Omega} \varphi\left(x,\left|D u_{n}(x)\right|\right)\left|D u_{n}(x)\right| d x}{\int_{\Omega}\left|D u_{n}(x)\right| d x} \leq K_{0}
$$

Since $\Phi \in N(\Omega)$, there exists $R>0$ such that

$$
\inf _{x \in \Omega} \varphi(x, R) \geq \inf _{x \in \Omega} \frac{\Phi(x, R)}{R}>2 K_{0} .
$$

We define $\widetilde{\Omega}(R, n):=\left\{x \in \Omega|| D u_{n}(x) \mid \geq R\right\}$ and take for all $n$ with $\int_{\Omega}\left|D u_{n}(x)\right| d x \geq$ $2 R$ meas $\Omega$, then

$$
\begin{aligned}
& \frac{\int_{\Omega} \varphi\left(x,\left|D u_{n}(x)\right|\right)\left|D u_{n}(x)\right| d x}{\int_{\Omega}\left|D u_{n}(x)\right| d x} \\
& \quad \geq \inf _{x \in \Omega} \varphi(x, R) \frac{\int_{\widetilde{\Omega}(R, n)}\left|D u_{n}(x)\right| d x}{\int_{\widetilde{\Omega}(R, n)}\left|D u_{n}(x)\right| d x+R \cdot \operatorname{meas}(\Omega)} \\
& \quad \geq \frac{1}{2} \inf _{x \in \Omega} \varphi(x, R)>K_{0} .
\end{aligned}
$$

This is a contradiction, thus (2.12) holds.

Lemma 2.2 (see [20], Remark 2.1) Let $V$ be a vector space of finite dimension and $A: V \rightarrow$ $V^{\prime}$ be a continuous mapping with

$$
\lim _{\|u\|_{V} \rightarrow+\infty} \frac{(A(u), u)}{\|u\|_{V}}=+\infty
$$

where $V^{\prime}$ is the dual space of $V$, then $A$ is surjective.

Lemma 2.3 (see [21], Lemma 2.1) If $u \in W^{1} L_{\Phi}(\Omega)$, then $u^{+}, u^{-} \in W^{1} L_{\Phi}(\Omega)$, and

$$
D u^{+}=\left\{\begin{array}{ll}
D u, & \text { if } u>0, \\
0, & \text { if } u \leq 0,
\end{array} \text { and } D u^{-}= \begin{cases}0, & \text { if } u \geq 0 \\
-D u, & \text { if } u<0\end{cases}\right.
$$

Here $u^{+}=\max \{u, 0\}, u^{-}=-\min \{u, 0\}$. This lemma holds in $W_{0}^{1} L_{\Phi}(\Omega)$ as well.

Lemma 2.4 (see [17]) If a sequence $g_{n} \in L_{\bar{\Phi}}(\Omega)$ converges in measure to a measurable function $g$ and if $g_{n}$ remains bounded in $L_{\bar{\Phi}}(\Omega)$, then $g \in L_{\bar{\Phi}}(\Omega)$ and $g_{n} \rightarrow g$ for $\sigma\left(L_{\bar{\Phi}}(\Omega), E_{\Phi}(\Omega)\right)$.

The following propositions refer to Theorems 1.6-1.8 in [16], Theorem 4.2 in [22], and Theorem 2.1 in [18].

Proposition 2.1 Let $\Phi \in N(\Omega)$ and

$$
\Phi_{1}(x, u)=a \Phi(x, b u) \quad(a, b>0), \text { for all } u \geq 0, x \in \Omega .
$$


Then $\Phi_{1} \in N(\Omega)$ and the complementary function $\overline{\Phi_{1}}$ to $\Phi_{1}$ is given by

$$
\overline{\Phi_{1}}(x, v)=a \bar{\Phi}\left(x, \frac{v}{a b}\right), \quad \text { for all } v \geq 0, x \in \Omega
$$

where $\bar{\Phi}$ is the complementary function to $\Phi$.

Proof It is easy to see that $\Phi_{1} \in N(\Omega)$. We only need to show (2.14). By (2.1) and (2.13), we can deduce that

$$
\varphi_{1}(x, \tau)=a b \varphi(x, b \tau), \quad \text { for all } \tau \geq 0, x \in \Omega,
$$

where $\varphi$ and $\varphi_{1}$ are the right-hand derivatives of $\Phi$ and $\Phi_{1}$, respectively.

From (2.3), $\phi_{1}(x, \sigma)=\frac{1}{b} \sup \left\{b \tau: \varphi(x, b \tau) \leq \frac{\sigma}{a b}\right\}=\frac{1}{b} \phi\left(x, \frac{\sigma}{a b}\right), \forall \sigma \geq 0$ and $x \in \Omega$.

For $\forall v \geq 0$, by (2.2), $\bar{\Phi}_{1}(x, v)=a \int_{0}^{v} \phi\left(x, \frac{\sigma}{a b}\right) d \frac{\sigma}{a b}, \forall v \geq 0$ and $x \in \Omega$. Define $s=\frac{\sigma}{a b}$. Then $\bar{\Phi}_{1}(x, v)=a \int_{0}^{\frac{v}{a b}} \phi(x, s) d s=a \bar{\Phi}\left(x, \frac{v}{a b}\right), \forall v \geq 0$ and $x \in \Omega$.

Proposition 2.2 Let $\Phi_{1}, \Phi_{2} \in N(\Omega)$ and

$$
\Phi_{1}(x, u) \leq \Phi_{2}(x, u)+h(x), \quad \text { for some } h \in L^{1}(\Omega), \text { all } u \geq 0 \text { and } x \in \Omega .
$$

Then

$$
\overline{\Phi_{2}}(x, v) \leq \overline{\Phi_{1}}(x, v)+h(x), \quad \text { for all } v \geq 0 \text { and } x \in \Omega,
$$

where $\overline{\Phi_{1}}$ and $\overline{\Phi_{2}}$ are the complementary functions to $\Phi_{1}$ and $\Phi_{2}$, respectively.

Proof By (2.5) and (2.6), one has $\Phi_{2}\left(x, \phi_{2}(x, v)\right)+\overline{\Phi_{2}}(x, v)=\phi_{2}(x, v) \cdot v \leq \Phi_{1}\left(x, \phi_{2}(x, v)\right)+$ $\overline{\Phi_{1}}(x, v), \forall v \geq 0$ and $x \in \Omega$.

In view of (2.15), $\Phi_{2}\left(x, \phi_{2}(x, v)\right)+h(x) \geq \Phi_{1}\left(x, \phi_{2}(x, v)\right), \forall v \geq 0$ and $x \in \Omega$. Therefore, $\overline{\Phi_{2}}(x, v) \leq \overline{\Phi_{1}}(x, v)+h(x), \forall v \geq 0$ and $x \in \Omega$.

Proposition 2.3 Let $\Phi \in N(\Omega)$ and its complementary function is $\bar{\Phi}$. $\varphi$ and $\phi$ are given by (2.1) and (2.2), respectively. Then the following assertions are equivalent.

(1) $\Phi \in \Delta_{2}$.

(2) $\forall l_{1}>1$, there exist $K^{\prime}>1$ and a nonnegative function $\tilde{h}_{1} \in L^{1}(\Omega)$ such that

$$
\Phi\left(x, l_{1} u\right) \leq K^{\prime} \Phi(x, u)+\tilde{h}_{1}(x), \quad \text { for all } u \geq 0 \text { and a.e. } x \in \Omega \text {. }
$$

(3) $\forall l_{2}>1$, there exist $\varepsilon \in(0,1)$ and a nonnegative function $\tilde{h}_{2} \in L^{1}(\Omega)$ such that

$$
\Phi(x,(1+\varepsilon) u) \leq l_{2} \Phi(x, u)+\tilde{h}_{2}(x), \quad \text { for all } u \geq 0 \text { and a.e. } x \in \Omega
$$

(4) $\forall l_{3}>1$, there exist $\delta>0$ and a nonnegative function $\tilde{h}_{3} \in L^{1}(\Omega)$ such that

$$
\left(l_{3}+\delta\right) \bar{\Phi}(x, v) \leq \bar{\Phi}\left(x, l_{3} v\right)+\tilde{h}_{3}(x), \quad \text { for all } v \geq 0 \text { and a.e. } x \in \Omega
$$


(5) $\forall l_{4}>1$, there exist $l_{0}>1$ and a nonnegative function $\tilde{h}_{4} \in L^{1}(\Omega)$ such that

$$
\bar{\Phi}(x, v) \leq \frac{1}{l_{0} l_{4}} \bar{\Phi}\left(x, l_{4} v\right)+\tilde{h}_{4}(x), \quad \text { for all } v \geq 0 \text { and a.e. } x \in \Omega \text {. }
$$

(6) There exist $l_{5}>1$ and a nonnegative function $\tilde{h}_{5} \in L^{1}(\Omega)$ such that

$$
\bar{\Phi}(x, v) \leq \frac{1}{2 l_{5}} \bar{\Phi}\left(x, l_{5} v\right)+\tilde{h}_{5}(x), \quad \text { for all } v \geq 0 \text { and a.e. } x \in \Omega
$$

(7) There exist $l_{6}>0$ and a nonnegative function $\tilde{h}_{6} \in L^{1}(\Omega)$ such that

$$
u \varphi(x, 2 u) \leq l_{6} u \varphi(x, u)+\tilde{h}_{6}(x), \quad \text { for all } u \geq 0 \text { and a.e. } x \in \Omega \text {. }
$$

(8) $\forall m_{1}>1$, there exist $l_{7}>0$ and a nonnegative function $\tilde{h}_{7} \in L^{1}(\Omega)$ such that

$$
u \varphi\left(x, m_{1} u\right) \leq l_{7} u \varphi(x, u)+\tilde{h}_{7}(x), \quad \text { for all } u \geq 0 \text { and a.e. } x \in \Omega .
$$

Proof (1) $\Rightarrow(2)$. Since $\Phi \in \Delta_{2}$, by (2.7), there exist $K>1$ and a nonnegative function $h \in$ $L^{1}(\Omega)$ such that $\Phi(x, 2 u) \leq K \Phi(x, u)+h(x), \forall u \geq 0$ and a.e. $x \in \Omega$. For every $l_{1}>1$, there exists $n \in \mathbb{N}$ such that $2^{n} \geq l_{1}$. Then

$$
\begin{aligned}
\Phi\left(x, l_{1} u\right) & \leq \Phi\left(x, 2^{n} u\right) \leq K \Phi\left(x, 2^{n-1} u\right)+h(x) \\
& \leq K^{2} \Phi\left(x, 2^{n-2} u\right)+(K+1) h(x) \\
& \leq \cdots \leq K^{n} \Phi(x, u)+\left(K^{n-1}+\cdots+K+1\right) h(x) \\
& =K^{n} \Phi(x, u)+\frac{K^{n}-1}{K-1} h(x),
\end{aligned}
$$

$\forall u \geq 0$ and a.e. $x \in \Omega$. Taking $K^{\prime}=K^{n}$ and $\tilde{h}_{1}=\frac{K^{n}-1}{K-1} h(x)$, we can deduce the assertion (2).

$(2) \Rightarrow(3)$. For every $l_{2}>1$, by the assertion (2), there exist $K^{\prime}>l_{2}$ and a nonnegative function $\tilde{h}_{1} \in L^{1}(\Omega)$ such that

$$
\Phi(x, 2 u) \leq K^{\prime} \Phi(x, u)+\tilde{h}_{1}(x), \quad \text { for all } u \geq 0 \text { and a.e. } x \in \Omega
$$

Take $\varepsilon=\frac{l_{2}-1}{K^{\prime}-1}$, then $\varepsilon \in(0,1)$. Hence,

$$
\begin{aligned}
\Phi(x,(1+\varepsilon) u) & =\Phi(x,(1-\varepsilon) u+2 \varepsilon u) \leq(1-\varepsilon) \Phi(x, u)+\varepsilon \Phi(x, 2 u) \\
& \leq(1-\varepsilon) \Phi(x, u)+K^{\prime} \varepsilon \Phi(x, u)+\varepsilon \tilde{h}_{1}(x)=l_{2} \Phi(x, u)+\varepsilon \tilde{h}_{1}(x)
\end{aligned}
$$

for all $u \geq 0$ and a.e. $x \in \Omega$. Taking $\tilde{h}_{2}=\varepsilon \tilde{h}_{1}$, we complete the assertion (3).

$(3) \Rightarrow(4)$. By the assertion (3), $\forall l_{3}>1$, there exist $\varepsilon \in(0,1)$ and a nonnegative function $\tilde{h}_{2} \in L^{1}(\Omega)$ such that

$$
\Phi(x,(1+\varepsilon) u) \leq l_{3} \Phi(x, u)+\tilde{h}_{2}(x), \quad \text { for all } u \geq 0 \text { and a.e. } x \in \Omega \text {. }
$$


It implies that $\frac{1}{l_{3}} \Phi(x,(1+\varepsilon) u) \leq \Phi(x, u)+\frac{1}{l_{3}} \tilde{h}_{2}(x)$. Denote $\Phi_{1}(x, u)=\frac{1}{l_{3}} \Phi(x,(1+\varepsilon) u)$. By Proposition 2.1, $\overline{\Phi_{1}}(x, v)=\frac{1}{l_{3}} \bar{\Phi}\left(x, \frac{l_{3}}{1+\varepsilon} v\right), \forall v \geq 0$ and a.e. $x \in \Omega$. By Proposition 2.2, we get

$$
\overline{\Phi_{1}}(x, v) \leq \frac{1}{l_{3}} \bar{\Phi}\left(x, \frac{l_{3}}{1+\varepsilon} v\right)+\frac{1}{l_{3}} \tilde{h}_{2}(x) \leq \frac{1}{l_{3}(1+\varepsilon)} \bar{\Phi}\left(x, l_{3} v\right)+\frac{1}{l_{3}} \tilde{h}_{2}(x),
$$

$\forall v \geq 0$, and a.e. $x \in \Omega$. Thus, we have $l_{3}(1+\varepsilon) \overline{\Phi_{1}}(x, v) \leq \bar{\Phi}\left(x, l_{3} v\right)+(1+\varepsilon) \tilde{h}_{2}(x)$. Taking $\delta=l_{3} \varepsilon$ and $\tilde{h}_{3}=(1+\varepsilon) \tilde{h}_{2}$, we complete the assertion (4).

$(4) \Rightarrow(5)$. By the assertion (4), $\forall l_{4}>1$, there exist $\delta>0$ and a nonnegative function $\tilde{h}_{3} \in$ $L^{1}(\Omega)$ such that

$$
\left(l_{4}+\delta\right) \bar{\Phi}(x, v) \leq \bar{\Phi}\left(x, l_{4} v\right)+\tilde{h}_{3}(x), \quad \forall v \geq 0 \text { and a.e. } x \in \Omega
$$

Hence, $\Phi(x, v) \leq \frac{1}{l_{4}\left(1+\frac{\delta}{l_{4}}\right)} \bar{\Phi}\left(x, l_{4} v\right)+\frac{1}{l_{4}\left(1+\frac{\delta}{l_{4}}\right)} \tilde{h}_{3}(x)$. Taking $l_{0}=1+\frac{\delta}{l_{4}}$ and $\tilde{h}_{4}=\frac{1}{l_{4}\left(1+\frac{\delta}{l_{4}}\right)} \tilde{h}_{3}$, we complete the assertion (5).

$(5) \Rightarrow(1)$. By the assertion (5), $\forall l_{4}>1$, there exist $l_{0}>1$ and a nonnegative function $\tilde{h}_{4} \in$ $L^{1}(\Omega)$ such that

$$
\bar{\Phi}(x, v) \leq \frac{1}{l_{0} l_{4}} \bar{\Phi}\left(x, l_{4} v\right)+\tilde{h}_{4}(x), \quad \forall v \geq 0 \text { and a.e. } x \in \Omega .
$$

By Proposition 2.1 and Proposition 2.2, we obtain $\Phi\left(x, l_{0} u\right) \leq l_{0} l_{4} \Phi(x, u)+l_{0} l_{4} \tilde{h}_{4}(x), \forall u \geq 0$ and a.e. $x \in \Omega$. Take $n_{0} \in \mathbb{N}$ such that $l_{0}^{n_{0}} \geq 2$. Then $\Phi(x, 2 u) \leq \Phi\left(x, l_{0}^{n_{0}} u\right) \leq l_{0}^{n_{0}} l_{4}^{n_{0}} \Phi(x, u)+$ $\frac{l_{0}^{n_{0}} l_{4}^{n_{0}}-1}{l_{0} l_{4}-1} \tilde{h}_{4}(x)$. Denote $l_{0}^{n_{0}} l_{4}^{n_{0}}=K$ and $\frac{l_{0}^{n_{0}} l_{0}^{n_{0}}-1}{l_{0} l_{4}-1} \tilde{h}_{4}=h$. We deduce (2.7), i.e. $\Phi \in \Delta_{2}$.

$(6) \Rightarrow(1)$. Define $\Psi_{1}(x, v)=\frac{1}{2 l_{5}} \bar{\Phi}\left(x, l_{5} v\right)$. By Proposition 2.1, $\Psi_{1}(x, u)=\frac{1}{2 l_{5}} \Phi(x, 2 u), \forall u \geq 0$ and a.e. $x \in \Omega$. By Proposition 2.2, $\Phi(x, 2 u) \leq 2 l_{5} \Phi(x, u)+2 l_{5} \tilde{h}_{5}(x), \forall u \geq 0$ and a.e. $x \in \Omega$. Therefore, $\Phi \in \Delta_{2}$.

Similarly, (1) implies (6).

$(1) \Rightarrow(7)$. By (2), there exist $K^{\prime}>0$ and $\tilde{h}_{1} \in L^{1}(\Omega)$ such that

$$
\Phi(x, 4 u) \leq K^{\prime} \Phi(x, u)+\tilde{h}_{1}(x), \quad \text { for all } u \geq 0 \text { and a.e. } x \in \Omega \text {. }
$$

On the other hand, we have $2 u \varphi(x, 2 u) \leq \Phi(x, 4 u)$ and $\Phi(x, u) \leq u \varphi(x, u)$, for $x \in \Omega, u \geq 0$. Hence,

$$
u \varphi(x, 2 u) \leq \frac{K^{\prime}}{2} u \varphi(x, u)+\frac{1}{2} \tilde{h}_{1}(x), \quad \text { for all } u \geq 0 \text { and a.e. } x \in \Omega
$$

Consequently, the assertion (7) holds by taking $l_{6}=\frac{K^{\prime}}{2}$ and $\tilde{h}_{6}=\frac{1}{2} \tilde{h}_{1}$.

(7) $\Rightarrow(8)$. For every $m_{1}>1$, there is $n_{0} \in \mathbb{N}^{+}$such that $2^{n_{0}} \geq m_{1}$. Then $u \varphi\left(x, m_{1} u\right) \leq$ $u \varphi\left(x, 2^{n_{0}} u\right) \leq l_{6}^{n_{0}} u \varphi(x, u)+\frac{l_{6}^{n_{0}}-1}{l_{6}-1} \tilde{h}_{6}(x), \forall u \geq 0$ and a.e. $x \in \Omega$. Taking $l_{7}=l_{6}^{n_{0}}$ and $\tilde{h}_{7}=$ $\frac{l_{6}^{n_{0}}-1}{l_{6}-1} \tilde{h}_{6}$, we complete $(8)$.

$(8) \Rightarrow(1)$. For every $l_{1}>1$, we have $\Phi\left(x, l_{1} u\right) \leq l_{1} u \varphi\left(x, l_{1} u\right)$. By (8), there exist $l_{7}>0$ and $\tilde{h}_{7} \in L^{1}(\Omega)$ such that

$$
u \varphi\left(x, l_{1} u\right) \leq l_{7} u \varphi\left(x, \frac{u}{2}\right)+\tilde{h}_{7}(x), \quad \text { for all } u \geq 0 \text { and a.e. } x \in \Omega \text {. }
$$


It follows that $\Phi\left(x, l_{1} u\right) \leq l_{1} l_{7} u \varphi\left(x, \frac{u}{2}\right)+l_{1} \tilde{h}_{7}(x) \leq 2 l_{1} l_{7} \Phi(x, u)+l_{1} \tilde{h}_{7}(x)$, for all $u \geq 0$ and a.e. $x \in \Omega$. Taking $K^{\prime}=2 l_{1} l_{7}$ and $\tilde{h}_{1}=l_{1} \tilde{h}_{7}$, we deduce (2). Immediately, (1) holds.

Example 2.1 Let $\Phi(x,|t|)=\left(1+\frac{|t|}{p(x)}\right) \ln \left(1+\frac{|t|}{p(x)}\right)-\frac{|t|}{p(x)}$, for $x \in \Omega$ and $t \in \mathbb{R}$, where $p: \Omega \rightarrow \mathbb{R}$ is a measurable function such that $1<p_{-} \leq p(x) \leq p_{+}<+\infty$. Then $\varphi(x,|t|)=\frac{1}{p(x)} \ln \left(1+\frac{|t|}{p(x)}\right)$, $\phi(x,|s|)=p(x)(\exp (p(x)|s|)-1)$ and $\Phi(x,|s|)=\exp (p(x)|s|)-p(x)|s|-1$. It follows that $\Phi \in$ $N(\Omega)$ and $\Phi \in \Delta_{2}$. But $\bar{\Phi} \notin \Delta_{2}$. Moreover, both $\Phi$ and $\Phi$ are locally integrable. Therefore, $L_{\Phi}(\Omega)$ is separable, but $L_{\Phi}(\Omega)$ is not reflexive.

Remark 2.1 Let $\Phi(x,|t|)=\exp (p(x)|t|)-1$, for $x \in \Omega$ and $t \in \mathbb{R}$, where $p: \Omega \rightarrow \mathbb{R}$ is a measurable function such that $1<p_{-} \leq p(x) \leq p_{+}<+\infty$. It is worth noting that $\Phi$ does not satisfy the condition $\lim _{u \rightarrow 0^{+}} \sup _{x \in \Omega} \frac{\Phi(x, u)}{u}=0$. Therefore, $\Phi \notin N(\Omega)$.

Clearly, by (2.9), Proposition 2.1 and Proposition 2.2, we can deduce the following proposition.

Proposition 2.4 If $\Phi \preceq \Psi$, then $\bar{\Psi} \preceq$.

\section{Existence theorems}

Let $\Phi \in N(\Omega)$, and satisfy the condition

( $\Phi) ~ \Phi \in \Delta_{2}, \bar{\Phi}$ is a complementary function to $\Phi$, both $\Phi$ and $\Phi$ are locally integrable and satisfy $\left(\Phi_{2}\right)$.

We assume that there exists $\Psi \in N(\Omega)$ satisfying the condition

( $\Psi) ~ \Psi \in \Delta_{2}, \bar{\Psi}$ is a complementary function to $\Psi$, both $\Psi$ and $\bar{\Psi}$ are locally integrable and satisfy $\left(\Phi_{2}\right), \Phi \preceq \Psi$, and the embedding $W^{1} L_{\Phi}(\Omega) \hookrightarrow L_{\Psi}(\Omega)$ is compact.

Note that, in this case, the spaces $L_{\Phi}(\Omega), L_{\Psi}(\Omega), W^{1} L_{\Phi}(\Omega), W_{0}^{1} L_{\Phi}(\Omega)$ are separable Banach spaces.

For $u, v \in L^{0}(\Omega)$, we denote $u \wedge v=\min \{u, v\}, u \vee v=\max \{u, v\}, u^{+}:=u \vee 0, u^{-}:=-u \wedge 0$, $u \leq v \Leftrightarrow u(x) \leq v(x)$ for a.e. $x \in \Omega$.

Let $a_{1}: \Omega \times \mathbb{R}^{N} \rightarrow \mathbb{R}^{N}$ be a Carathéodory function satisfying the following conditions:

$\left(A_{1}\right)$ For a.e. $x \in \Omega$ and all $\xi, \eta \in \mathbb{R}^{N}$,

$$
\begin{aligned}
& \left|a_{1}(x, \xi)\right| \leq b_{1} \bar{\Phi}^{-1}(x, \Phi(x,|\xi|))+g_{1}(x), \\
& a_{1}(x, \xi) \xi \geq b_{2} \Phi(x,|\xi|)-g_{2}(x), \\
& {\left[a_{1}(x, \xi)-a_{1}(x, \eta)\right](\xi-\eta)>0, \quad \xi \neq \eta,}
\end{aligned}
$$

where $b_{1}, b_{2}>0, g_{1} \in E_{\bar{\Phi}}(\Omega), g_{1} \geq 0, g_{2} \in L^{1}(\Omega)$, and $g_{2} \geq 0$.

Let $a_{0}: \Omega \times \mathbb{R} \rightarrow \mathbb{R}$ be a Carathéodory function satisfying the following conditions:

$\left(A_{0}\right)$ For a.e. $x \in \Omega$ and all $t \in \mathbb{R}$,

$$
\left|a_{0}(x, t)\right| \leq b_{1} \bar{\Phi}^{-1}(x, \Phi(x,|t|))+g_{1}(x)
$$

where $b_{1}>0, g_{1} \in E_{\bar{\Phi}}(\Omega)$, and $g_{1} \geq 0$. 


\section{Example 3.1}

(1) Let $\Phi(x,|t|)=\frac{1}{p(x)}|t|^{p(x)}, a_{1}(x, \xi)=|\xi|^{p(x)-2} \xi$, for $x \in \Omega$ and $t \in \mathbb{R}$, where $p: \Omega \rightarrow \mathbb{R}$ is a measurable function such that $2 \leq p_{-} \leq p(x) \leq p_{+}<+\infty$. Then $\Phi$ satisfies $(\Phi)$ and we get the $p(x)$-Laplace operator $\operatorname{div}\left(|D u|^{p(x)-2} D u\right)$.

(2) Let $\Phi(x,|t|)=\frac{1}{p(x)}\left[\left(1+|t|^{2}\right)^{p(x) / 2}-1\right], a_{1}(x, \xi)=\left(1+|\xi|^{2}\right)^{(p(x)-2) / 2} \xi$, for $x \in \Omega$ and $t \in \mathbb{R}$, where $p: \Omega \rightarrow \mathbb{R}$ is a measurable function such that $2 \leq p_{-} \leq p(x) \leq p_{+}<+\infty$. Then $\Phi$ satisfies $(\Phi)$ and we obtain the generalized mean curvature operator $\operatorname{div}\left(\left(1+|D u|^{2}\right)^{(p(x)-2) / 2} D u\right)$. Moreover, by Proposition 2.3(6), $\bar{\Phi} \in \Delta_{2}$.

(3) Let $\Phi(x,|t|)=\left(1+\frac{|t|}{p(x)}\right) \ln \left(1+\frac{|t|}{p(x)}\right)-\frac{|t|}{p(x)}$, for $x \in \Omega$ and $t \in \mathbb{R}$, where $p: \Omega \rightarrow \mathbb{R}$ is a measurable function such that $1<p_{-} \leq p(x) \leq p_{+}<+\infty$. Clearly, it can be verified that $\Phi$ satisfies $(\Phi)$. Put $a_{1}(x, \xi)=\varphi(x,|\xi|) \frac{\xi}{|\xi|}$, and $a_{0}(x, t)=\varphi(x,|t|)$, for $x \in \Omega, t \in \mathbb{R}$ and $\xi \in \mathbb{R}^{N}$, where $\varphi(x,|t|)=\frac{1}{p(x)} \ln \left(1+\frac{|t|}{p(x)}\right)$. Then $a_{1}$ and $a_{0}$ satisfy $\left(A_{1}\right)$ and $\left(A_{0}\right)$, respectively.

Remark 3.1 Clearly, the condition (1.2) (resp. (1.5)) implies (3.1) (resp. (3.4)).

Consider the following Dirichlet boundary value problem:

$$
\left\{\begin{array}{l}
-\operatorname{div}\left(a_{1}(x, D u)\right)+a_{0}(x, u)=f(x, u, D u), \quad \text { in } \Omega, \\
u=0, \quad \text { on } \partial \Omega,
\end{array}\right.
$$

where $f: \Omega \times \mathbb{R} \times \mathbb{R}^{N} \rightarrow \mathbb{R}$ is a Carathéodory function. Denote by $F$ the Nemytskii operator associated to $f$, that is,

$$
F(u)(x)=f(x, u(x), D u(x)), \quad \text { for } x \in \Omega .
$$

A function $u$ is called a (weak) solution of (3.5) if $u \in W_{0}^{1} L_{\Phi}(\Omega), F(u) \in L_{\bar{\Psi}}(\Omega)$ and $u$ satisfies the equation

$$
\int_{\Omega} a_{1}(x, D u) D v d x+\int_{\Omega} a_{0}(x, u) v d x=\int_{\Omega} f(x, u, D u) v d x, \quad \text { for all } v \in W_{0}^{1} L_{\Phi}(\Omega) .
$$

A function $u$ is called a subsolution (resp. supersolution) of (3.5) if $u \in W_{0}^{1} L_{\Phi}(\Omega), F(u) \in$ $L_{\bar{\Psi}}(\Omega)$ and (3.6) holds with '=' replaced by ' $\leq$ ' (resp. ' $\geq$ ') for every nonnegative functions $v$ in $W_{0}^{1} L_{\Phi}(\Omega)$ (see [2]).

Theorem 3.1 Suppose that $\underline{u}_{1}, \ldots, \underline{u}_{k}$ and $\bar{u}_{1}, \ldots, \bar{u}_{m}$ are subsolutions and supersolutions of (3.5), respectively, that satisfy

$$
\underline{u}:=\underline{u}_{1} \vee \underline{u}_{2} \vee \cdots \vee \underline{u}_{k} \leq \bar{u}_{1} \wedge \bar{u}_{2} \wedge \cdots \wedge \bar{u}_{m}:=\bar{u} .
$$

Let $(\Phi),(\Psi),\left(A_{1}\right),\left(A_{0}\right)$ hold. Assume the nonlinear term $g$ satisfies the following local growth condition:

$$
|f(x, t, \xi)| \leq q(x)+b_{3} \bar{\Phi}^{-1}(x, \Phi(x,|t|))+b_{4} \bar{\Psi}^{-1}(x, \Phi(x,|\xi|)),
$$

for a.e. $x \in \Omega$ and $\forall t \in[\underline{u}(x), \bar{u}(x)]$, with $q \in E_{\bar{\Psi}}(\Omega), b_{3}, b_{4}>0$. Then there exists a solution $u$ of (3.5) such that $\underline{u} \leq u \leq \bar{u}$. 
Proof Denote $V=W_{0}^{1} L_{\Phi}(\Omega)$. For $x \in \Omega$, we put

$$
T u(x)= \begin{cases}\bar{u}(x), & \text { if } u(x)>\bar{u}(x), \\ u(x), & \text { if } \underline{u}(x) \leq u(x) \leq \bar{u}(x), \quad \text { for } u \in V . \\ \underline{u}(x), & \text { if } u(x)<\underline{u}(x),\end{cases}
$$

Then $T u=u \vee \underline{u}+u \wedge \bar{u}-u$. By Remark 3.1 in [2], $T: V \rightarrow V$ is continuous. It is easy to see that $T$ is bounded. From Proposition 2.4, we obtain $F(T u) \in L_{\bar{\Psi}}(\Omega), \forall u \in V$.

We define the cutoff function $l: \Omega \times \mathbb{R} \rightarrow \mathbb{R}$ given by

$$
l(x, s)= \begin{cases}\bar{\Phi}^{-1}(x, \Phi(x, s-\bar{u}(x))), & \text { if } s>\bar{u}(x), \\ 0, & \text { if } \underline{u}(x) \leq s \leq \bar{u}(x), \\ -\bar{\Phi}^{-1}(x, \Phi(x, \underline{u}(x)-s)), & \text { if } s<\underline{u}(x),\end{cases}
$$

for $x \in \Omega, s \in \mathbb{R}$. Then $l$ satisfies the following condition:

$$
|l(x, s)| \leq \bar{\Phi}^{-1}(x, \Phi(x, 2|s|))+\bar{\Phi}^{-1}(x, \Phi(x, 2|\bar{u}(x)|))+\bar{\Phi}^{-1}(x, \Phi(x, 2|\underline{u}(x)|)),
$$

for $x \in \Omega$ and all $s \in \mathbb{R}$.

For all $u \in V$, since $\Phi \in \Delta_{2}$, there exists $K_{1}>1$ such that

$$
\begin{aligned}
& \int_{\Omega} l(x, u) u d x \\
& =\int_{\{u>\bar{u}\}} \bar{\Phi}^{-1}(x, \Phi(x, u-\bar{u}))(u-\bar{u}) d x \\
& +\int_{\{u>\bar{u}\}} \bar{\Phi}^{-1}(x, \Phi(x, u-\bar{u})) \bar{u} d x \\
& +\int_{\{u<\underline{u}\}} \bar{\Phi}^{-1}(x, \Phi(x, \underline{u}-u))(\underline{u}-u) d x \\
& -\int_{\{u<\underline{u}\}} \bar{\Phi}^{-1}(x, \Phi(x, \underline{u}-u)) \underline{u} d x \\
& \geq \int_{\{u>\bar{u}\}} \Phi(x, u-\bar{u}) d x-\int_{\{u>\bar{u}\}}\left[\frac{1}{2} \Phi(x, u-\bar{u})+\Phi(x, 2|\bar{u}|)\right] d x \\
& +\int_{\{u<\underline{u}\}} \Phi(x, \underline{u}-u) d x-\int_{\{u<\underline{u}\}}\left[\frac{1}{2} \Phi(x, \underline{u}-u)+\Phi(x, 2|\underline{u}|)\right] d x \\
& =\frac{1}{2} \int_{\{u>\bar{u}\}} \Phi(x, u-\bar{u}) d x-\int_{\{u>\bar{u}\}} \Phi(x, 2|\bar{u}|) d x \\
& +\frac{1}{2} \int_{\{u<\underline{u}\}} \Phi(x, \underline{u}-u) d x-\int_{\{u<\underline{u}\}} \Phi(x, 2|\underline{u}|) d x \\
& \geq \frac{1}{2} \int_{\{u>\bar{u}\}}\left[2 \Phi\left(x, \frac{|u|}{2}\right)-\Phi(x,|\bar{u}|)\right] d x-\int_{\Omega} \Phi(x, 2|\bar{u}|) d x \\
& +\frac{1}{2} \int_{\{u<\underline{u}\}}\left[2 \Phi\left(x, \frac{|u|}{2}\right)-\Phi(x,|\underline{u}|)\right] d x \\
& -\int_{\Omega} \Phi(x, 2|\underline{u}|) d x
\end{aligned}
$$




$$
\begin{aligned}
\geq & \int_{\{u>\bar{u}\} \cup\{u<\underline{u}\}} \Phi\left(x, \frac{|u|}{2}\right) d x-C \\
& +\int_{\{\bar{u} \leq u \leq \underline{u}\}}\left[\Phi\left(x, \frac{|u|}{2}\right)-\Phi\left(x, \frac{|\bar{u}| \vee|\underline{u}|}{2}\right)\right] d x \\
= & \int_{\Omega} \Phi\left(x, \frac{|u|}{2}\right) d x-C \\
\geq & \frac{1}{K_{1}} \int_{\Omega} \Phi(x,|u|) d x-C,
\end{aligned}
$$

for some constant $C>0$ independent of $u$, where $\{u<\underline{u}\}=\{x \in \Omega: u(x)<\underline{u}(x)\},\{u>\bar{u}\}=$ $\{x \in \Omega: u(x)>\bar{u}(x)\}$, and $\{\underline{u} \leq u \leq \bar{u}\}=\{x \in \Omega: \underline{u}(x) \leq u(x) \leq \bar{u}(x)\}$.

Let us consider the auxiliary equation of finding $u \in V$ such that

$$
\begin{gathered}
\int_{\Omega} a_{1}(x, D u) D v d x+\int_{\Omega} a_{0}(x, T u) v d x+\lambda \int_{\Omega} l(x, u) v d x \\
=\int_{\Omega} F(T u) v d x, \quad \forall v \in V
\end{gathered}
$$

where $\lambda>0$ is a parameter to be specified later.

Define $\Gamma_{T}: V \rightarrow V^{*}$,

$$
\left(\Gamma_{T} u, v\right):=\int_{\Omega} a_{1}(x, D u) D v d x+\int_{\Omega} a_{0}(x, T u) v d x+\lambda \int_{\Omega} l(x, u) v d x-\int_{\Omega} F(T u) v d x,
$$

$\forall v \in V$. Then $\Gamma_{T}$ is well defined.

Since $\Phi \in \Delta_{2}$, there exists a sequence $\left\{w_{n}\right\} \subset V$ such that $\left\{w_{n}\right\}$ is dense in $V$. Let $V_{m}=$ $\operatorname{span}\left\{w_{1}, \ldots, w_{m}\right\}$ and consider $\left.\Gamma_{T}\right|_{V_{m}}$. For every $u \in V_{m},\|D u\|_{\Phi}$ and $\int_{\Omega}|D u| d x$ are two norms of $V_{m}$ equivalent to the usual norm of finite dimensional vector spaces.

Similar to the proof of Proposition 3.1 in [20], we can deduce that the mapping $u \rightarrow$ $\left.\Gamma_{T}\right|_{V_{m}} u: V_{m} \rightarrow V_{m}^{*}$ is continuous.

In view of (3.7), one has

$$
\begin{aligned}
& \left|\int_{\Omega} F(T u) u d x\right| \\
& \leq C^{*}\|q\|_{\Psi}\|u\|_{1, \Phi}+2 b_{3} \int_{\Omega} \Phi(x,|u|) d x+b_{3} \int_{\Omega} \Phi(x,|\bar{u}|) d x+b_{3} \int_{\Omega} \Phi(x,|\underline{u}|) d x \\
& \quad+b_{4} \varepsilon_{1} \int_{\Omega} \Phi(x,|D u|) d x+b_{4} \int_{\Omega} \varepsilon_{1} \Psi\left(x, \frac{1}{\varepsilon_{1}}|\bar{u}| \vee|\underline{u}|\right) d x+b_{4} \int_{\Omega} \Psi(x,|\bar{u}|) d x \\
& \quad+b_{4} \int_{\Omega} \Psi(x,|\underline{u}|) d x+b_{4} \int_{\Omega} \Phi(x,|D \bar{u}|) d x+b_{4} \int_{\Omega} \Phi(x,|D \underline{u}|) d x
\end{aligned}
$$

for all $u \in V$, where $\varepsilon_{1}=\frac{b_{2}}{2 b_{4}}$ and the constant $C^{*}>0$.

Thanks to (3.4) and (2.8), there exist $K_{2}>1$ and a nonnegative function $h \in L^{1}(\Omega)$ such that

$$
\begin{aligned}
& \left|\int_{\Omega} a_{0}(x, T u) u d x\right| \\
& \quad \leq b_{1}\left(K_{2}-1\right) \int_{\Omega}[\Phi(x,|u|)+\Phi(x,|\underline{u}|)+\Phi(x,|\bar{u}|)] d x+b_{1} \int_{\Omega} h(x) d x
\end{aligned}
$$




$$
\begin{aligned}
& +\left(b_{1}+1\right) \int_{\Omega} \Phi(x,|u|) d x+\int_{\Omega} \Phi\left(x,\left|g_{1}(x)\right|\right) d x \\
= & \left(b_{1} K_{2}+1\right) \int_{\Omega} \Phi(x,|u|) d x+C,
\end{aligned}
$$

for all $u \in V$, where the constant $C>0$ is independent of $u$.

Let $\lambda>K_{1}\left(b_{1} K_{2}+1+2 b_{3}\right)$. Combining (3.2), (3.9), (3.11), and (3.12), we obtain

$$
\begin{aligned}
\left(\Gamma_{T} u, u\right) \geq & \frac{b_{2}}{2} \int_{\Omega} \Phi(x,|D u|) d x+\left(\frac{\lambda}{K_{1}}-b_{1} K_{2}-1-2 b_{3}\right) \int_{\Omega} \Phi(x,|u|) d x \\
& -C-C^{*}\|q\|_{\bar{\Psi}}\|u\|_{1, \Phi} \\
\geq & \frac{b_{2}}{2} \int_{\Omega} \Phi(x,|D u|) d x-C-C^{*}\|q\|_{\bar{\Psi}}\|u\|_{1, \Phi},
\end{aligned}
$$

for all $u \in V$ and some $C>0$ independent of $u$. By Proposition 1.9 in [2], there exists $C_{1}>0$ such that $\|u\|_{\Phi} \leq C_{1}\|D u\|_{\Phi}$. In view of (3.13), for all $u \in V_{m}$, we have

$$
\begin{aligned}
\frac{\left(\left.\Gamma_{T}\right|_{V_{m}} u, u\right)}{\|u\|_{1, \Phi}} & \geq \frac{b_{2} \int_{\Omega} \Phi(x,|D u|) d x}{2\left(1+C_{1}\right)\|D u\|_{\Phi}}-\frac{C}{\|u\|_{1, \Phi}}-C^{*}\|q\|_{\Psi} \\
& \geq \frac{b_{2} \int_{\Omega} \Phi(x,|D u|) d x}{2 C_{2}\left(1+C_{1}\right) \int_{\Omega}|D u| d x}-\frac{C}{\|u\|_{1, \Phi}}-C^{*}\|q\|_{\bar{\Psi}}
\end{aligned}
$$

for some constant $C_{2}>0$. By Lemma 2.1, we get

$$
\frac{\left(\left.\Gamma_{T}\right|_{V_{m}} u, u\right)}{\|u\|_{1, \Phi}} \rightarrow+\infty, \quad \text { as }\|u\|_{1, \Phi} \rightarrow+\infty
$$

By Lemma 2.2, there exists a Galerkin solution $u_{m} \in V_{m}$ for every $m \in \mathbb{N}$ such that $\left(\Gamma_{T} u_{m}, v\right)=0, v \in V_{m}$. Using the density of $\left\{w_{m}\right\}$, we deduce that

$$
\left(\Gamma_{T} u_{m}, v\right)=0, \quad \forall v \in V .
$$

For $u \in V$, define $\rho(u)=\int_{\Omega}(\Phi(x,|D u|)+\Phi(x,|u|)) d x$ and $\|u\|_{\rho}=\inf \left\{\lambda>0: \rho\left(\frac{u}{\lambda}\right) \leq 1\right\}$. Then $\|u\|_{\rho}$ is a norm of $V$ equivalent to $\|u\|_{1, \Phi}$ (see [2]).

Taking $\alpha_{0}=\min \left\{\frac{b_{2}}{2}, \frac{\lambda}{K_{1}}-b_{1} K_{2}-1-2 b_{3}\right\}$, we have

$$
\begin{aligned}
&\left(\Gamma_{T} u, u\right) \geq \alpha_{0}\left[\int_{\Omega} \Phi(x,|D u|) d x+\int_{\Omega} \Phi(x,|u|) d x\right]-C-C^{*}\|q\|_{\bar{\Psi}}\|u\|_{1, \Phi} \\
& \geq \alpha_{0}\left(\|u\|_{\rho}-\varepsilon\right)\left[\int_{\Omega} \Phi\left(x, \frac{|D u|}{\|u\|_{\rho}-\varepsilon}\right) d x+\int_{\Omega} \Phi\left(x, \frac{|u|}{\|u\|_{\rho}-\varepsilon}\right) d x\right] \\
&-C-C^{*}\|q\|_{\bar{\Psi}}\|u\|_{1, \Phi} \\
& \geq \alpha_{0}\left(\|u\|_{\rho}-\varepsilon\right)-C-C^{*}\|q\|_{\bar{\Psi}}\|u\|_{1, \Phi},
\end{aligned}
$$

for all $u \in V$, as $\|u\|_{1, \Phi}$ is large enough. Therefore, by (3.15), we get a sequence $\left\{u_{m}\right\}$ that is bounded in $V$. Hence, there exist $u_{0} \in V$ and a subsequence $\left\{u_{k}\right\}$ of $\left\{u_{m}\right\}$, such 
that

$$
\begin{array}{ll}
u_{k} \rightarrow u_{0} & \text { weakly in } V \text { for } \sigma\left(\prod L_{\Phi}, \prod E_{\bar{\Phi}}\right), \\
u_{k} \rightarrow u_{0} & \text { strongly in } L_{\Psi}(\Omega), \\
u_{k} \rightarrow u_{0} & \text { a.e. in } \Omega,
\end{array}
$$

as $k \rightarrow \infty$.

By (3.4) and (3.8), $\left\{a_{0}\left(x, T u_{k}\right)\right\}$ and $\left\{l\left(x, u_{k}\right)\right\}$ are bounded in $L_{\bar{\Phi}}(\Omega)$. By Lemma 2.4,

$$
a_{0}\left(x, T u_{k}\right) \rightarrow a_{0}\left(x, T u_{0}\right) \quad \text { weakly in } L_{\bar{\Phi}}(\Omega) \text { for } \sigma\left(L_{\bar{\Phi}}, E_{\Phi}\right)
$$

and

$$
l\left(x, u_{k}\right) \rightarrow l\left(x, u_{0}\right) \quad \text { weakly in } L_{\bar{\Phi}}(\Omega) \text { for } \sigma\left(L_{\bar{\Phi}}, E_{\Phi}\right)
$$

as $k \rightarrow \infty$.

On the other hand, by the Lebesgue theorem, we deduce that

$$
\int_{\Omega} a_{0}\left(x, T u_{k}\right)\left(u_{k}-u_{0}\right) d x \rightarrow 0, \quad \int_{\Omega} l\left(x, u_{k}\right)\left(u_{k}-u_{0}\right) d x \rightarrow 0, \quad \text { as } k \rightarrow \infty .
$$

Thanks to (3.7), $\left\{F\left(T u_{k}\right)\right\}$ is bounded in $L_{\bar{\Psi}}(\Omega)$. Hence,

$$
\int_{\Omega} F\left(T u_{k}\right)\left(u_{k}-u_{0}\right) d x \rightarrow 0, \quad \text { as } k \rightarrow \infty
$$

Thus we obtain

$$
\int_{\Omega} a_{1}\left(x, D u_{k}\right)\left(D u_{k}-D u_{0}\right) d x \rightarrow 0, \quad \text { as } k \rightarrow \infty
$$

Similar to the proof of Proposition 3.1 in [20], we can construct a subsequence still denoted by $\left\{u_{k}\right\}$ such that

$$
D u_{k} \rightarrow D u_{0} \quad \text { a.e. in } \Omega \text {, as } k \rightarrow \infty \text {. }
$$

Hence

$$
a_{1}\left(x, D u_{k}\right) \rightarrow a_{1}\left(x, D u_{0}\right) \quad \text { a.e. in } \Omega \text {, as } k \rightarrow \infty \text {. }
$$

In view of (3.1), $\left\{a_{1}\left(x, D u_{k}\right)\right\}$ is bounded in $\left(L_{\bar{\Phi}}(\Omega)\right)^{N}$, then by Lemma 2.4 , we have

$$
a_{1}\left(x, D u_{k}\right) \rightarrow a_{1}\left(x, D u_{0}\right) \quad \text { weakly in }\left(L_{\bar{\Phi}}(\Omega)\right)^{N} \text { for } \sigma\left(\left(L_{\bar{\Phi}}(\Omega)\right)^{N},\left(E_{\Phi}(\Omega)\right)^{N}\right)
$$

as $k \rightarrow \infty$. Similarly,

$$
F\left(T u_{k}\right) \rightarrow F\left(T u_{0}\right) \quad \text { weakly in } L_{\bar{\Psi}}(\Omega) \text { for } \sigma\left(L_{\bar{\Psi}}, E_{\Psi}\right) \text {, as } k \rightarrow \infty \text {. }
$$


Hence, $\left(\Gamma_{T} u_{k}, v\right)=\left(\Gamma_{T} u_{0}, v\right), \forall v \in V$. By (3.15), $\left(\Gamma_{T} u_{0}, v\right)=0, \forall v \in V$, i.e., $u_{0}$ is a solution of (3.10).

For every $m \in \mathbb{N}$, taking $v=\left(u_{m}-\bar{u}\right)^{+} \in V$ in (3.15) as a test function, we get

$$
\begin{aligned}
\int_{\Omega} & {\left[a_{1}\left(x, D u_{m}\right)-a_{1}(x, D \bar{u})\right] D\left(u_{m}-\bar{u}\right)^{+} d x } \\
& +\int_{\Omega}\left[a_{0}\left(x, T u_{m}\right)-a_{0}(x, \bar{u})\right]\left(u_{m}-\bar{u}\right)^{+} d x+\lambda \int_{\Omega} l\left(x, u_{m}\right)\left(u_{m}-\bar{u}\right)^{+} d x \\
\leq & \int_{\Omega}\left[F\left(T u_{m}\right)-F(\bar{u})\right]\left(u_{m}-\bar{u}\right)^{+} d x .
\end{aligned}
$$

By (3.3), we have

$$
\begin{aligned}
& \int_{\Omega} {\left[a_{1}\left(x, D u_{m}\right)-a_{1}(x, D \bar{u})\right] D\left(u_{m}-\bar{u}\right)^{+} d x } \\
&=\int_{\left\{u_{m}>\bar{u}\right\}}\left[a_{1}\left(x, D u_{m}\right)-a_{1}(x, D \bar{u})\right] D\left(u_{m}-\bar{u}\right) d x \geq 0 .
\end{aligned}
$$

Since

$$
\int_{\Omega}\left[a_{0}\left(x, T u_{m}\right)-a_{0}(x, \bar{u})\right]\left(u_{m}-\bar{u}\right)^{+} d x=0
$$

and

$$
\int_{\Omega}\left[F\left(T u_{m}\right)-F(\bar{u})\right]\left(u_{m}-\bar{u}\right)^{+} d x=0,
$$

we get

$$
0 \geq \int_{\Omega} l\left(x, u_{m}\right)\left(u_{m}-\bar{u}\right)^{+} d x \geq \int_{\left\{u_{m}>\bar{u}\right\}} \Phi\left(x, u_{m}-\bar{u}\right) d x \geq 0 .
$$

It follows that $u_{m} \leq \bar{u}$. Using arguments similar to those above we can prove that $u_{m} \geq \underline{u}$. Thanks to (3.18), one has $\underline{u} \leq u_{0} \leq \bar{u}$. From the definitions of $l\left(\cdot, u_{0}(\cdot)\right)$ and $T u_{0}$, we have

$$
l\left(x, u_{0}(x)\right)=0, \quad a_{0}\left(x, T u_{0}(x)\right)=a_{0}\left(x, u_{0}(x)\right)
$$

and

$$
f\left(x, T u_{0}(x), D T u_{0}(x)\right)=f\left(x, u_{0}(x), D u_{0}(x)\right)
$$

for a.e. $x \in \Omega$. We note that then (3.10) reduces to (3.6), which completes the proof.

Remark 3.2 Our proof does not need the conditions $\bar{\Phi} \in \Delta_{2}$ and ( $\left.\Phi_{3}\right)$ in [2].

Remark 3.3 Our method needs the strict monotonicity (3.3) of $a_{1}$, but does not require monotonicity (1.7) or coercivity (1.6) of $a_{0}$. However, if $\bar{\Phi} \in \Delta_{2}$, then we can deduce (3.22) by following the lines of Theorem 4.1 in [23] when (3.3) is replaced by (1.4). 
Remark 3.4 Assume that (1.7) holds and the assumptions of Theorem 3.1 hold. If $f(x, u, D u)=f(x) \in L_{\bar{\Psi}}(\Omega)$, then it is easy to see that (3.5) has a unique solution.

Remark 3.5 Now we consider the following Neumann boundary value problem:

$$
\left\{\begin{array}{l}
-\operatorname{div}\left(a_{1}(x, D u)\right)+a_{0}(x, u)=f(x, u, D u), \quad \text { in } \Omega, \\
a_{1}(x, D u) \cdot \gamma=0, \quad \text { on } \partial \Omega
\end{array}\right.
$$

where $\gamma$ is the outward unit normal to $\partial \Omega$.

We also assume that there is a function $G:[k,+\infty) \rightarrow \mathbb{R}$ for some $k>0$ such that $G(s) \rightarrow$ $+\infty$ as $s \rightarrow+\infty$ and

$$
\Phi(x, s u) \geq G(s) s \Phi(x, u)-\operatorname{sh}(x), \quad \text { for all } s>0, u \geq 0 \text {, a.e. } x \in \Omega,
$$

and some $h \in L^{1}(\Omega), h \geq 0$.

Assume that (3.25) holds and the assumptions of Theorem 3.1 hold. Replacing $V$ by $W^{1} L_{\Phi}(\Omega)$ in the proof of Theorem 3.1, and (3.13)-(3.14) by the following lines, we can deduce a similar theorem to Theorem 3.1 for the Neumann boundary value problem (3.24).

$$
\begin{aligned}
\left(\Gamma_{T} u, u\right) \geq & \frac{b_{2}}{2} \int_{\Omega} \Phi(x,|D u|) d x+\left(\frac{\lambda}{K_{1}}-b_{1} K_{2}-1-2 b_{3}\right) \int_{\Omega} \Phi(x,|u|) d x \\
& -C-C^{*}\|q\|_{\Psi}\|u\|_{1, \Phi} \\
\geq & \alpha_{0}\left[\int_{\Omega} \Phi(x,|D u|) d x+\int_{\Omega} \Phi(x,|u|) d x\right]-C-C^{*}\|q\|_{\bar{\Psi}}\|u\|_{1, \Phi},
\end{aligned}
$$

for all $u \in V$ and some $C>0$ independent of $u$, where $\alpha_{0}=\min \left\{\frac{b_{2}}{2}, \frac{\lambda}{K_{1}}-b_{1} K_{2}-1-2 b_{3}\right\}$.

Combining (3.25) and (3.26), we can deduce that, for any $\varepsilon>0$,

$$
\begin{aligned}
& \left(\Gamma_{T} u, u\right) \\
& \geq \alpha_{0}\left[\int_{\Omega} \Phi\left(x,\left(\|u\|_{\rho}-\varepsilon\right) \frac{|D u|}{\|u\|_{\rho}-\varepsilon}\right) d x+\int_{\Omega} \Phi\left(x,\left(\|u\|_{\rho}-\varepsilon\right) \frac{|u|}{\|u\|_{\rho}-\varepsilon}\right) d x\right] \\
& \quad-C-C^{*}\|q\|_{\Psi}\|u\|_{1, \Phi} \\
& \geq \alpha_{0}\left(\|u\|_{\rho}-\varepsilon\right) G\left(\left(\|u\|_{\rho}-\varepsilon\right)\right)\left[\int_{\Omega} \Phi\left(x, \frac{|D u|}{\|u\|_{\rho}-\varepsilon}\right) d x+\int_{\Omega} \Phi\left(x, \frac{|u|}{\|u\|_{\rho}-\varepsilon}\right) d x\right] \\
& \quad-\alpha_{0}\left(\|u\|_{\rho}-\varepsilon\right) \int_{\Omega}|h(x)| d x-C-C^{*}\|q\|_{\bar{\Psi}}\|u\|_{1, \Phi} \\
& \geq \alpha_{0}\left(\|u\|_{\rho}-\varepsilon\right) G\left(\left(\|u\|_{\rho}-\varepsilon\right)\right)-\alpha_{0}\left(\|u\|_{\rho}-\varepsilon\right) \int_{\Omega}|h(x)| d x-C \\
& \quad-C^{*}\|q\|_{\bar{\Psi}}\|u\|_{1, \Phi},
\end{aligned}
$$

$\forall u \in V$, as $\|u\|_{1, \Phi}$ is large enough. Since $\varepsilon$ is arbitrary, we get

$$
\left(\Gamma_{T} u, u\right) \geq \alpha_{0}\|u\|_{\rho} G\left(\|u\|_{\rho}\right)-\alpha_{0}\|u\|_{\rho} \int_{\Omega}|h(x)| d x-C-C^{*}\|q\|_{\bar{\Psi}}\|u\|_{1, \Phi},
$$


$\forall u \in V$, as $\|u\|_{1, \Phi}$ is large enough. Therefore, we obtain

$$
\frac{\left(\left.\Gamma_{T}\right|_{V_{m}} u, u\right)}{\|u\|_{1, \Phi}} \rightarrow+\infty, \quad \text { as }\|u\|_{1, \Phi} \rightarrow+\infty
$$

Proposition 3.1 If $\bar{\Phi} \in \Delta_{2}$, then there are functions $h \in L^{1}(\Omega), h \geq 0$, and $G:[k,+\infty) \rightarrow \mathbb{R}$ for some $k>2$ such that $G(s) \rightarrow+\infty$ as $s \rightarrow+\infty$ and (3.25) holds.

Proof The proof of (3.25) is similar to the proof of Lemma 3.14 of [24].

Since $\bar{\Phi} \in \Delta_{2}$, there exist a positive constant $k>1$ and a nonnegative function $h \in L^{1}(\Omega)$ such that $\bar{\Phi}(x, 2 v) \leq k \bar{\Phi}(x, v)+h(x)$, for all $v \geq 0$ and a.e. $x \in \Omega$. Necessarily, $k>2$. Defining a function $F:[1,+\infty) \rightarrow[k,+\infty)$ by

$$
F(r)=r\left((1-\lambda) k^{n}+\lambda k^{n+1}\right) \quad \text { if } r \in\left[2^{n}, 2^{n+1}\right] \text { and } r=(1-\lambda) 2^{n}+\lambda 2^{n+1} \text {, }
$$

we obtain

$$
\begin{aligned}
\bar{\Phi}(x, r v) & \leq\left[(1-\lambda) k^{n}+\lambda k^{n+1}\right] \bar{\Phi}(x, v)+\left[(1-\lambda) \frac{k^{n}-1}{k-1}+\lambda \frac{k^{n+1}-1}{k-1}\right] h(x) \\
& \leq\left[(1-\lambda) k^{n}+\lambda k^{n+1}\right] \Phi(x, v)+\left[(1-\lambda) k^{n}+\lambda k^{n+1}\right] h(x) \\
& \leq F(r) \bar{\Phi}(x, v)+\frac{F(r)}{r} h(x) .
\end{aligned}
$$

Hence $\frac{1}{F(r)} \bar{\Phi}(x, r v) \leq \bar{\Phi}(x, v)+\frac{1}{r} h(x)$. Taking $\Psi_{1}(x, v)=\frac{1}{F(r)} \bar{\Phi}(x, r v)$, by Proposition 2.1 and Proposition 2.2, we have $\Phi(x, u) \leq \frac{1}{F(r)} \Phi\left(x, \frac{F(r)}{r} u\right)+\frac{1}{r} h(x)$, for all $u \geq 0$ and a.e. $x \in \Omega$. It follows that $F(r) \Phi(x, u) \leq \Phi\left(x, \frac{F(r)}{r} u\right)+\frac{F(r)}{r} h(x)$, for all $u \geq 0$ and a.e. $x \in \Omega$. Since $\frac{F(r)}{r}$ strictly increases from $k$ to $+\infty$ as $r \in[1,+\infty)$, its reciprocal function $G(s)$ is well defined and strictly increases from 1 to $+\infty$ as $s \in[k,+\infty)$, and we have $s G(s) \Phi(x, u) \leq \Phi(x, s u)+\operatorname{sh}(x)$, i.e.

$$
\Phi(x, s u) \geq s G(s) \Phi(x, u)-s h(x), \quad \text { for } s \geq k, u \geq 0 \text { and a.e. } x \in \Omega \text {. }
$$

Remark 3.6 Clearly, (1.9) can be replaced by (3.25) in the proof of Theorem 2.1 in [2]. Therefore, by Proposition 3.1, the condition (1.9) can be omitted since $\bar{\Phi} \in \Delta_{2}$ in [2].

Denote $\mathcal{S}=\left\{u \in W_{0}^{1} L_{\Phi}(\Omega): u\right.$ is a solution of (3.5) and $\left.\underline{u} \leq u \leq \bar{u}\right\}$. Under the assumptions of Theorem 3.1, the solution set $\mathcal{S}$ is nonempty and we can deduce the following corollary.

Corollary 3.1 Under the assumptions of Theorem 3.1, the following assertions about $\mathcal{S}$ are true.

(a) The set $\mathcal{S}$ is compact in $W_{0}^{1} L_{\Phi}(\Omega)$.

(b) $\mathcal{S}$ is a direct set in both directions, that is, if $u_{1}, u_{2} \in \mathcal{S}$ then there exist $u, v \in \mathcal{S}$ such that $u \geq u_{1} \vee u_{2}$ and $v \leq u_{1} \wedge u_{2}$.

(c) $\mathcal{S}$ has least and greatest elements with respect to the ordering ' $\leq$ ', that is, there are $u_{*}, u^{*} \in \mathcal{S}$ such that $u_{*} \leq u \leq u^{*}$, for all $u \in \mathcal{S}$. 


\section{Competing interests}

The authors declare that they have no competing interests.

\section{Authors' contributions}

All authors contributed equally and significantly in writing this paper. All authors read and approved the final manuscript.

\section{Author details}

'Department of Mathematics, Tongji University, Siping Road, Shanghai, 200092, China. ${ }^{2}$ Department of Basic Teaching, Shanghai Jian Qiao University, Huchenghuan Road 1111, Shanghai, 201306, China.

\section{Acknowledgements}

The authors are highly grateful for the referees' careful reading and comments on this paper. The first author was supported by 'Chen Guang' Project (supported by Shanghai Municipal Education Commission and Shanghai Education Development Foundation) (10CGB25). The second author was supported by the National Natural Science Foundation of China (11371279).

Received: 14 December 2015 Accepted: 12 May 2016 Published online: 31 May 2016

\section{References}

1. Le, V: On a sub-supersolution method for variational inequalities with Leray-Lions operators in variable exponent spaces. Nonlinear Anal. 71, 3305-3321 (2009)

2. Fan, X: Differential equations of divergence form in Musielak-Sobolev spaces and a sub-supersolution method. J. Math. Anal. Appl. 386, 593-604 (2012)

3. Liu, D, Zhao, P: Solutions for a quasilinear elliptic equation in Musielak-Sobolev spaces. Nonlinear Anal., Real World Appl. 26, 315-329 (2015)

4. Le, $\mathrm{V}$ : A range and existence theorem for pseudomonotone perturbations of maximal monotone operators. Proc. Am. Math. Soc. 139, 1645-1658 (2011)

5. Fu, Y, Shan, Y: Removable sets for Hölder continuous solutions of elliptic equations involving variable exponent. J. Math. Anal. Appl. 424, 1296-1322 (2015)

6. Rădulescu, V: Nonlinear elliptic equations with variable exponent: old and new. Nonlinear Anal. 121, 336-369 (2015)

7. Barletta, G, Chinnì, A, O'Regan, D: Existence results for a Neumann problem involving the $p(x)$-Laplacian with discontinuous nonlinearities. Nonlinear Anal., Real World Appl. 27, 312-325 (2016)

8. Dong, G, Shi, Z: An existence theorem for weak solutions for a class of elliptic partial differential systems in Orlicz spaces. Nonlinear Anal. 68, 1037-1042 (2008)

9. Dong, G: An existence theorem for weak solutions for a class of elliptic partial differential systems in general Orlicz-Sobolev spaces. Nonlinear Anal. 69, 2049-2057 (2008)

10. Dong, G: Elliptic equations with measure data in Orlicz spaces. Electron. J. Differ. Equ. 2008, 76 (2008)

11. Dong, G, Fang, $X$ : Variational inequalities with multivalued lower order terms and convex functionals in Orlicz-Sobolev spaces. J. Funct. Spaces (2015). doi:10.1155/2015/321437

12. Faria, L, Miyagaki, O, Motreanu, D, Tanaka, M: Existence results for nonlinear elliptic equations with Leray-Lions operator and dependence on the gradient. Nonlinear Anal. 96, 154-166 (2014)

13. Adams, R, Fournier, J: Sobolev Spaces, 2nd edn. Academic Press, New York (2003)

14. Kozlowski, W: Advancements in fixed point theory in modular function spaces. Arab. J. Math. 1, 477-494 (2012)

15. Musielak, J: Orlicz Spaces and Modular Spaces. Lecture Notes in Math. Springer, Berlin (1983)

16. Wu, C, Wang, T, Chen, S, Wang, Y: Geometry Theory of Orlicz Spaces. Harbin University of Technology Press, Harbin (1986) (in Chinese)

17. Benkirane, A, Sidi El Vally, M: An existence result for nonlinear elliptic equations in Musielak-Orlicz-Sobolev spaces. Bull. Belg. Math. Soc. Simon Stevin 20, 57-75 (2013)

18. Wu, C, Wang, T: Orlicz Space and Its Application. Heilongjiang Science and Technology Press, Harbin (1983) (in Chinese)

19. Landes, R: Quasilinear elliptic operators and weak solutions of the Euler equation. Manuscr. Math. 27, 47-72 (1979)

20. Dong, G, Fang, X: Existence results for some nonlinear elliptic equations with measure data in Orlicz-Sobolev spaces. Bound. Value Probl. (2015). doi:10.1186/s13661-014-0278-0

21. Rodrigues, J, Teymurazyan, R: On the two obstacles problem in Orlicz-Sobolev spaces and applications. Complex Var. Elliptic Equ. 56, 769-787 (2011)

22. Krasnoselskii, M, Rutickii, Y: Convex Functions and Orlicz Space. Noordhoff, Groningen (1961)

23. Gwiazda, P, Wittbold, P, Wróblewska, A, Zimmermann, A: Renormalized solutions of nonlinear elliptic problems in generalized Orlicz spaces. J. Differ. Equ. 253, 635-666 (2012)

24. Gossez, J: Nonlinear elliptic boundary value problems for equations with rapidly (or slowly) increasing coefficients. Trans. Am. Math. Soc. 190, 163-205 (1974) 